\title{
Rituximab (RTX) and Plasmapheresis in a Recurrent Segmental and Focal Glomerulosclerosis (FSGS) Case after Kidney Transplantation
}

\author{
Abdellaoui I*, Azzabi A, Sahtout W, Benaicha N, Guedri Y, Zallama D and Achour A \\ Nephrology, Dialysis and Transplantation Ward, Sahloul Hospital of Sousse, Tunisia
}

\begin{abstract}
A 21-year-old male patient whose primary kidney disease was focal segmental glomerulosclerosis (FSGS) received a living kidney transplant 7 years after starting hemodialysis (haploidentic from his mother). His kidney disease was primary recurrent FSGS treated since the age of four with corticosteroids and switching different options of Immunosuppressive treatment Calcineurin inhibitors and mycophenolate Mofetil). The transplantation induction protocol was Corticosteroid (CS) boli and Basiliximab without added preventive Therapeutic Plasma Exchange (TPE/ Plasmapheresis) sessions. Then for maintain, he took CS, Mycophenolate Mofetil and Cyclosporine A. After 9 liters of urine output at day zero after transplantation, he developed severe recurrence of proteinuria (up to $23 \mathrm{~g} / 24$ hat day 2) and anuria with creatinin level ascension to reach 500 micromol/l. The immunosuppressive treatment consisted of ten daily Plasmapheresis sessions, five intravenous doses of Rituximab (RTX-375/m2) $700 \mathrm{mg}$ at days 1, 5, 9, 13 and three boli of CS $(500 \mathrm{mg})$ in addition to Cyclosporine (oral). At hospital discharge (1 month), proteinuria increased below nephrotic range at day 14 and serum creatinine returned progressively to normal values. He underwent two other Plasmapheresis sessions (TPE) but the third was delayed because of technical problems. At the sixth month, urine output decreased and proteinuria rose again. It was a relapse. Histology examination showed recurrence signs and he underwent additional Plasmapheresis sessions, another RTX intravenous dose, $500 \mathrm{mg}$ of CS and switched to Tacrolimus. This strategy allowed obtaining sustained full remission of the proteinuria and excellent graft function, which persists over 9 months after transplantation. No notable adverse events related to RTX or TPE were observed. This case confirms that RTX associated with Plasmapheresis may be an effective treatment of recurrent Nephrotic Syndrom (NS) due to FSGS.
\end{abstract}

Keywords: Rituximab; Kidney; Transplantation; Hemodialysis

\section{Introduction}

Primary focal segmental glomerulosclerosis (FSGS) is a serious etiology of NS and end-stage renal disease (ESRD). The abnormality of the visceral podocytes in the glomeruli is the elementary lesion in this disease [1]. The recurrence of FSGS in renal allograft recipients is mainly a challenge to nephrologist $[1]$. The incidence of recurrence is evaluated between $20 \%$ and $30 \%$ in 2008 [1]. In 2018, the recurrence is evaluated up to $55 \%$ of patients after kidney transplantation and it leads to early graft loss according to a new study [2]. The recurrence of the original disease is the second most common cause of graft loss, after allograft rejection. Recurrent FSGS exposes to a negative outcome, with a hazard ratio of graft failure that is two-fold higher than other glomerulonephritis [3]. In a large study from the Australia and New Zealand Dialysis and Transplant Registry (ANZDATA), the incidence of loss of kidney graft at 10 years caused by recurrent FSGS was $12.7 \%$ (95\% CI 7.3-21.6) [4]. The risk is higher in patients with idiopathic FSGS, pediatric patients, and patients who show rapid disease progression to ESRD [2]. A protein was identified as a circulating factor and seems to be associated with recurrence of FSGS after transplantation [2]. Since there is a lack of controlled trials, the treatment of recurrent FSGS is inconsistent and highly empirical. Prophylactic and perioperative treatment with Plasmapheresis and the use of cyclosporine as immunosuppressive drug choice, represent the main cornerstones of fighting this danger. Also recently, protocols with Rituximab have shown promising results [4]. The Treatment protocols are various and no recommendations were published. But it seems that treatment with therapeutic plasma exchange (TPE) and rituximab RTX appears to be safe, well tolerated and effective in the management of patients with post-transplant recurrent FSGS according to many recent studies [5].

The aim of our study was to describe the efficacy and safety of therapeutic plasma exchange (TPE), and Rituximab (RTX), in the management of a graft recipient presenting FSGS recurrence immediately after transplantation.

\section{Case Report}

We describe in this manuscript the case of a male patient aged 21 years old. His medical history is detailed in Figure 1. He has no family history of kidney diseases. His parents are not relatives. He was diagnosed having a NS. First, he showed response to Cs but over the age of 4 years the NS becomes corticosteroids resistant. Unfortunately, he reached ESRD after multiple switching from immunosuppressive drug to another at the age of 14 and underwent hemodialysis until the age of 21 (Figure 1). He received a kidney graft from living donor (his mother sharing with him similar Human Leukocyte Antigens (HLA)) in February 2017. The transplantation induction protocol was corticosteroids (CS) boli and Basiliximab without added preventive Therapeutic Plasma Exchange (TPE) sessions, Then for maintain he took CS, Mycophenolate Mofetil (MMF) and Ciclosporine A (Cyclo A). After 9 liters of urine output at day zero after transplantation, he developed severe recurrence of proteinuria (up to $23 \mathrm{~g} / 24 \mathrm{~h}$ at day 4 )

*Corresponding author: Abdellaoui I, Nephrology, Dialysis and Transplantation Ward, Sahloul Hospital of Sousse, Tunisia, Tel: 0021655635905; E-mail: i.men.ab87@gmail.com

Received: June 10, 2019; Accepted: June 24, 2019; Published: June 29, 2018

Citation: Abdellaoui I, Azzabi A, Sahtout W, Benaicha N, Guedri Y, et al. (2019) Rituximab (RTX) and Plasmapheresis in a Recurrent Segmental and Focal Glomerulosclerosis (FSGS) Case after Kidney Transplantation. J Nephrol Ther 9: 333.

Copyright: (C) 2018 Abdellaoui I, et al. This is an open-access article distributed under the terms of the Creative Commons Attribution License, which permits unrestricted use, distribution, and reproduction in any medium, provided the original author and source are credited. 


\section{Patient's History}

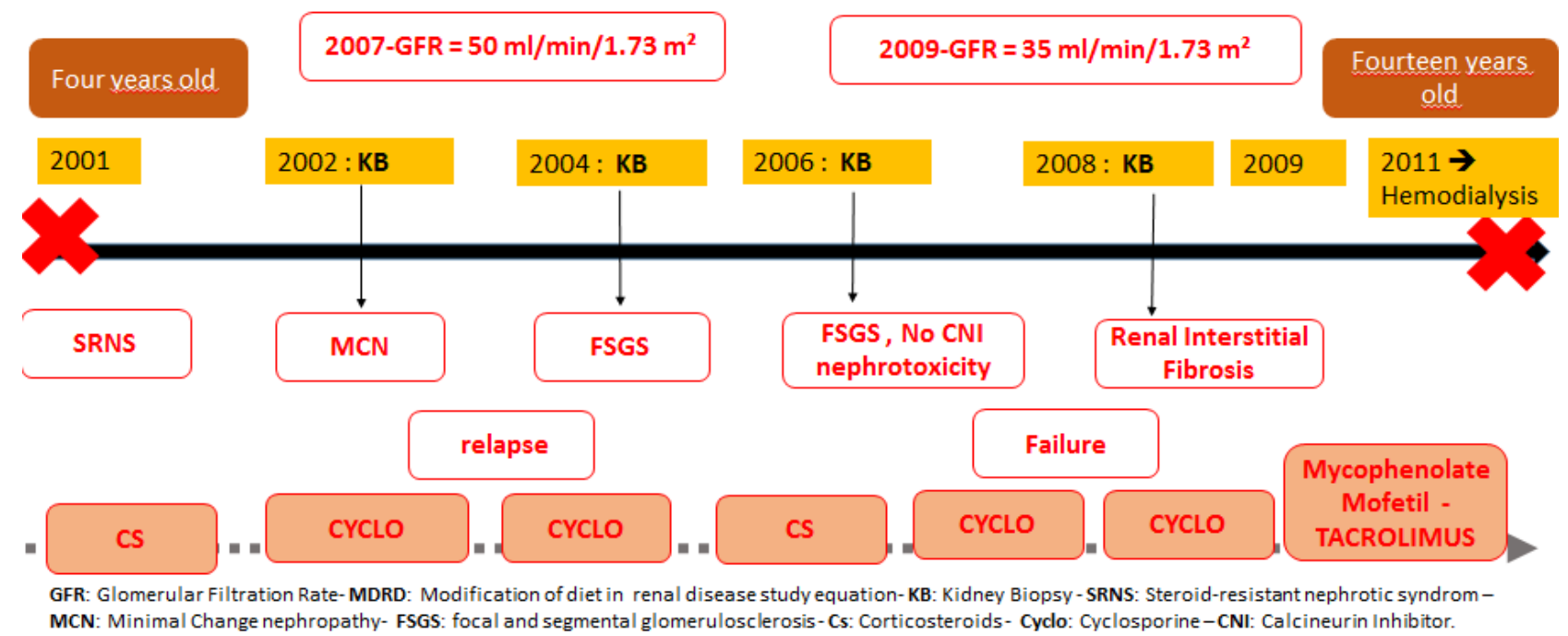

Figure 1: The medical renal history of the patient preceding the kidney transplantation.

and anuria with creatinin level ascension to 500 micromol at day Two. The graft morphological Ultrasound (US) and vessels Doppler US were normal. Thee graft biopsy was not performed for technical reasons but the discussion with the transplantation team concluded to a FSGS recurrence with clinical, biological and chronological arguments. The treatment was urgently applied the French Hospital "Necker" protocol for FSGS recurrence after transplantation was followed (Figure 2). The protocol was based on: more than $10 \mathrm{TPE}$ sessions + Rituximab (375 $\mathrm{mg} / \mathrm{m} 2)+$ Cs 500mg in boli + Cyclo A (Oral). Also he underwent initially a few hemodialysis sessions for the complications of anuria with a creatinin serum rate at $721 \mathrm{micromol} / \mathrm{l} /$. The graft function was ameliorated as the proteinuria rate decreased. At the first month M1 following transplantation, the creatinin rate was $97 \mathrm{micromol} / \mathrm{l}$ and the proteinuria was zero $\mathrm{g} / 24 \mathrm{~h}$. he was discharged at M1 and unfortunately, he missed one TPE session. He was stable until the $6^{\text {th }}$ month, when he got the same symptoms again: Oliguria and proteinuria discovered in urine strip test. Biology showed a serum creatinin rate at 315 micromol/l and proteinuria at $3.5 \mathrm{~g} / 24 \mathrm{~h}$. No hypertension or urinary tract bacterial infection, were found. The Cytotoxic antibodies test was negative, serum residual Cyclosporin rates were in range (150/900 ng/ $\mathrm{ml}$ ) and Jc/BK virus urinary tests were negative. After checking that the graft and its vessels were normal in Ultrasound test, we performed the graft biopsy. Results are detailed in Figures 3-5. No signs for CNI toxicity, acute allograft rejection or acute tubular necrosis were found. The diagnosis seems to be a relapse of FSGS and the treatment was as follows: 3 Corticosteroid 500 milligram intravenous boli + Plasmapheresis sessions + switch from Cyclo A to Tacrolimus. The patient responded well to the treatment. At the $9^{\text {th }}$ month following transplantation, he has a proteinuria rate at $0.14 \mathrm{~g} / 24 \mathrm{H}$ and e serum creatinin rate at $109 \mathrm{micromol} / \mathrm{l}$.

\section{Discussion}

In the literature, many cases of recurrence of FSGS in the first days after transplantation were published were similar to the patient described in this manuscript [6,7]. The proteinuria and the alteration in graft function appeared since day 2 after receiving the kidney graft. Two patterns of clinical presentations are observed: Early recurrence, which is characterized by massive proteinuria within hours to days after implantation of the renal graft, and late recurrence, which occurs several months or years after the transplantation [7]. The disappearance of all clinical and biological signs after Plasmapheresis is an argument for recurrent FSGS in our patient's case (No earl graft biopsy performed). Studies confirmed the existence of a protein described as a circulating permeability factors plays a crucial role in podocytes lesions and the progression of FSGS [8]. It was found that a serum soluble urokinase receptor (suPAR) is elevated in two-thirds of subjects with primary FSGS, but not in people with other glomerular diseases. We further find that a higher concentration of suPAR before transplantation underlies an increased risk for recurrence of FSGS after transplantation. It was shown that the he circulating suPAR activates podocyte's $\beta(3)$ integrin in both native and grafted kidneys, causing foot process effacement, proteinuria and FSGS-like glomerulopathy [8]. SuPAR involvement in the pathophysiology of the disease is well detailed, but its role as the only circulating factor remains debated. Moreover, the suPAR rate is not specific to the primary FSGS. S. Beaudreuil \& al has described in 2019 another molecule as a soluble circulating factor for FSGS called "CASK" [9]. CASK is a calcium calmoduline serine threonine kinase expressed by podocytes and is proved to have as a target the CD98 [9]. Delville \& al described a co-factor associated to suPAR, the CD-40 that is an autoantibody targeting glomerular antigens [10].

In the case of this patient, he missed a plas1mapheresis session and he showed lately a relapse. This rebound effect in serum suPAR rates in case of a lag in TPE sessions was described in some studies. Relapse can occur during rapid Plasmapheresis sessions and usually requires prolonged reinstitution of sessions [5]. Other risk factors for relapse were discussed in literature such as: young age when the 
Citation: Abdellaoui I, Azzabi A, Sahtout W, Benaicha N, Guedri Y, et al. (2019) Rituximab (RTX) and Plasmapheresis in a Recurrent Segmental and Focal Glomerulosclerosis (FSGS) Case after Kidney Transplantation. J Nephrol Ther 9: 333.

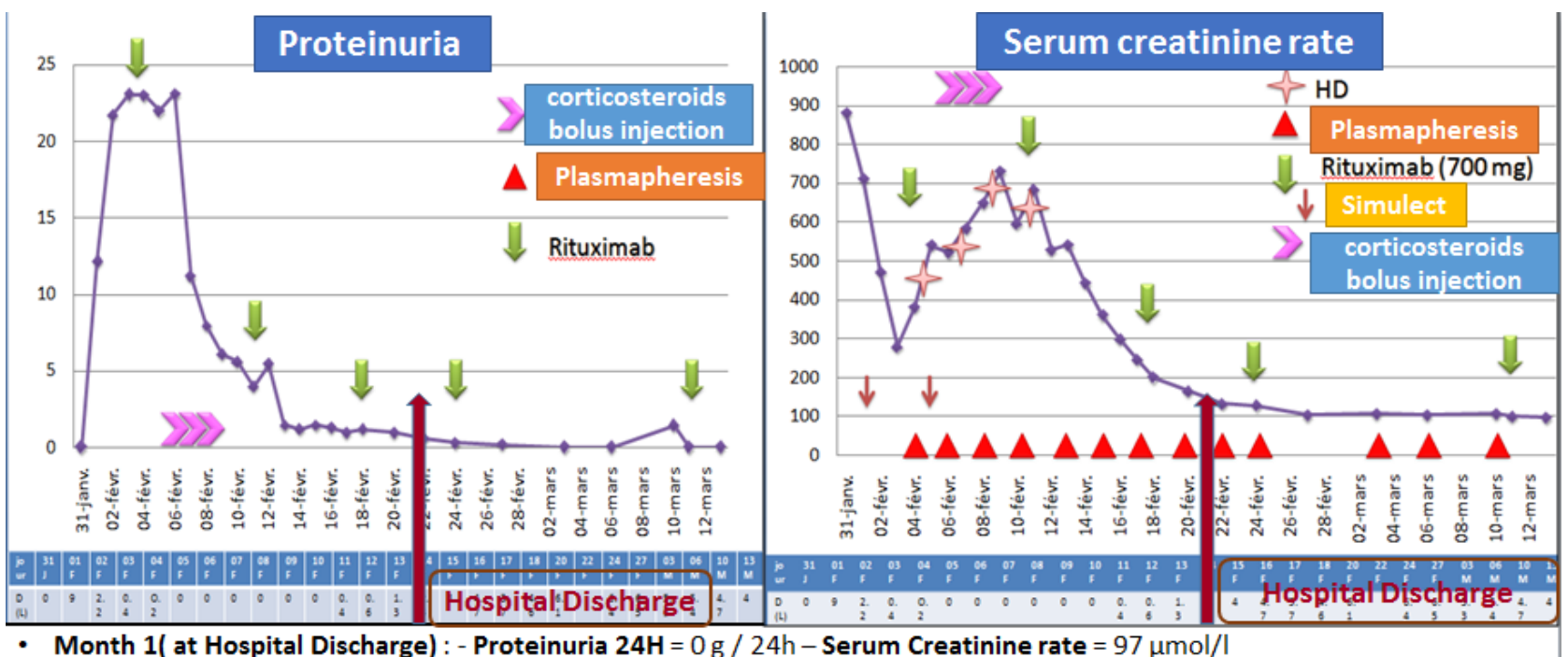

Figure 2: Immediate recurrence of FSGS following kidney transplantation and the patient outcomes after treatment.

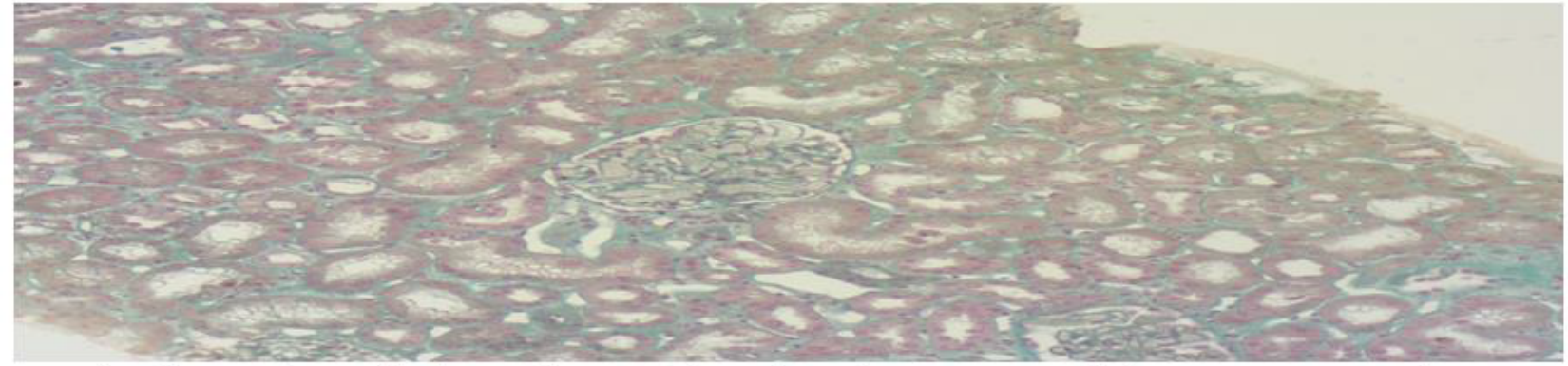

Masson's trichrome coloration (*40)- Normal aspect of glomeruli, no Fibrosis, no Acute Tubular Necrosis, no CNI nephrotoxicity.

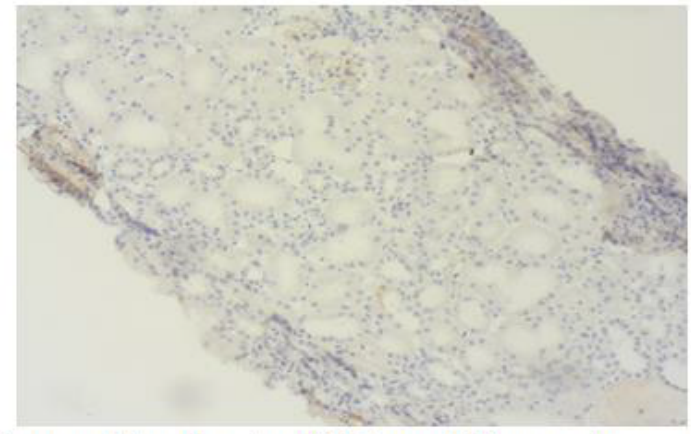

Immunohistochemistry (IHC) test: CD4 =negative

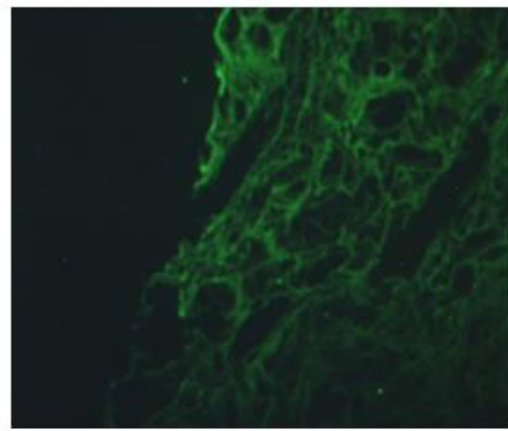

Direct flourescent antibody (DFA) test: CD4 (left) and IgG (right) negative

Figure 3: Results of the graft biopsy [Optic microscope*40-IHC test: CD4 negative-DFA test negative]

primary FSGS appeared ( $<16$ years), recurrence of FSGS after a first kidney graft, a rapid progression to ESRD (less than 3 years), ESRD at age less than 12 years, related living donor, HLA mismatches, donor's age more than 40 years and white race [5,11]. In this case, the patient has as risk factors: the age when the NS started was 4 years, the donor was his mother with no mismatches, first kidney graft but ESRD reached quickly in 10 years. The recurrent FSGS histological type according to Columbia classification can predict the prognosis $[12,13]$. The Columbia classification has been modified to characterize in a better way HSF lesions and their topography $\mathrm{n}$ the glomeruli and the nature of associated endocapillary and extracapillary alterations in order to ameliorate treatment [14]. It helps in graft FSGS by predicting relapse and précising the lesions types but its role is limited [14] the most frequent lesions in early recurrent NS after transplantation is "Minimal Change Disease" (MCD) and the characteristic lesions of FSGS can be observed lately. After transplantation, all histological types according to Columbia can be expected, every type can relapse and the histological lesions are not always the same of initial lesions of the native kidneys [13]. A multicenter study that included 21 cases of FSGS recurrence in kidney allografts showed histological similarity between native and allograft lesions in $81 \%$ of cases. The Cellular and collapsing lesions are not exchanged; they recur in their native forms 
Citation: Abdellaoui I, Azzabi A, Sahtout W, Benaicha N, Guedri Y, et al. (2019) Rituximab (RTX) and Plasmapheresis in a Recurrent Segmental and Focal Glomerulosclerosis (FSGS) Case after Kidney Transplantation. J Nephrol Ther 9: 333.

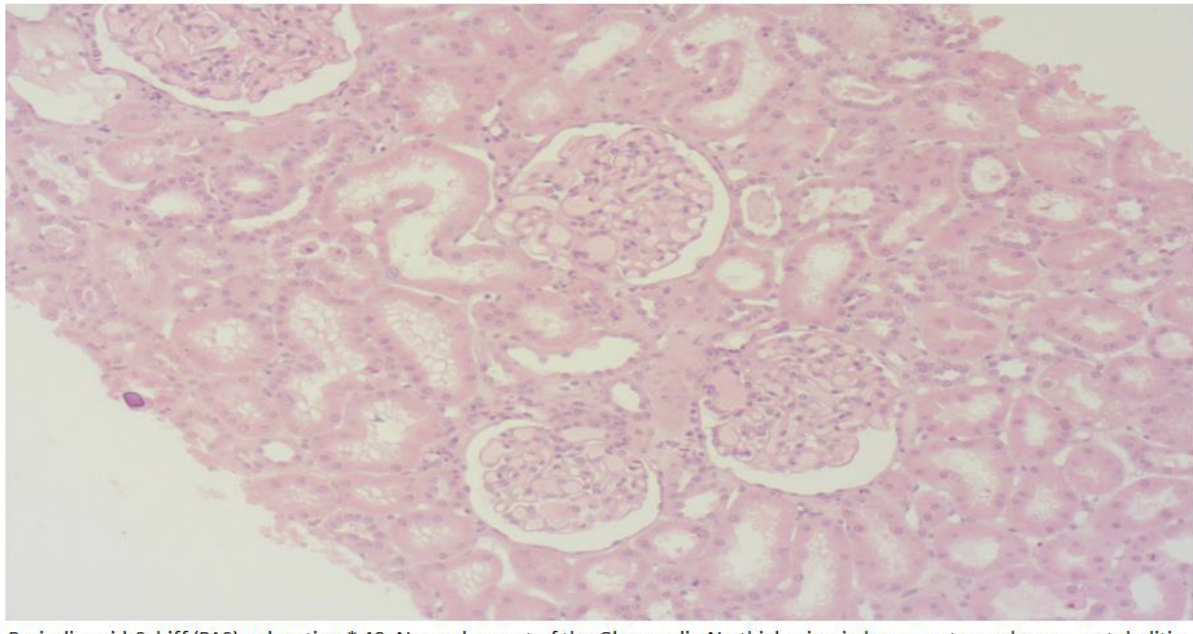

Periodic acid-Schiff (PAS) coloration * 40: Normal aspect of the Glomeruli= No thickening in basement membrane, no tubulitis.

Figure 4: Results of the graft biopsy [Optic microscope ${ }^{*} 40$ with PAS coloration showing the absence of Acute tubular necrosis].

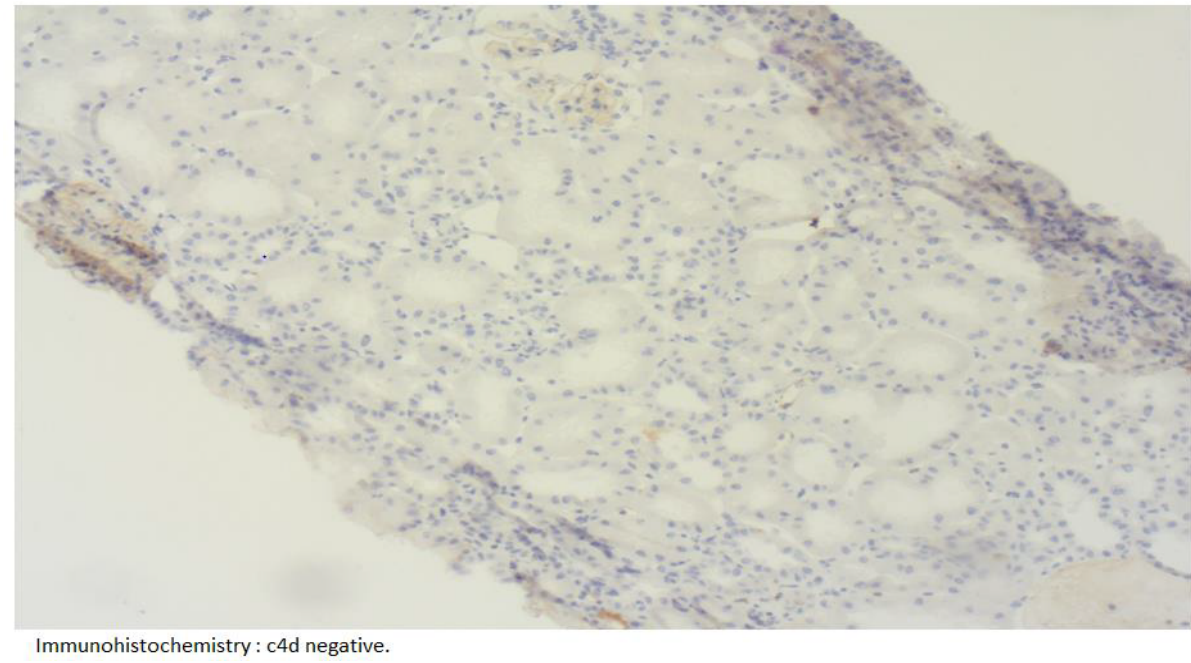

Figure 5: Results of the graft biopsy [IHC test CD4 negative-CD4-mediated allograft rejection=absent].

in the renal transplant [14]. Type I recurs with the same lesions, type II recurs with initial MCD lesions then FSGS but type3 recurs generally with initial different lesions [15].

There are not yet recommendations for the treatment of FSGS recurrence. Since there is a lack of controlled trials, the treatment of recurrent FSGS is empirical. Prophylactic and perioperative treatment with Plasmapheresis and the use of cyclosporine as immunosuppressive drug choice, represent the main cornerstones. Also recently, protocols with Rituximab has shown promising results [4]. In this systematic review of patients with recurrent post-transplant FSGS, 71\% of patients achieved full or partial remission after treatment with plasma exchange; however, extensive missing data and lack of a control group limit any conclusions on causality [16]. Kashgary \& al found in a systemic revue including 77 patients having recurrence of FSGS that $71 \%$ of patients achieved partial or complete remission after Plasmapheresis sessions [16]. A recent meta-analysis published in 2015 included six non-randomized studies with 117 transplantations. The primary outcome was the effect of Plasmapheresis in the achievement of a complete or partial remission. The results were confirming the role of Plasmapheresis in a better graft survival and in the achievement of remission [17]. According to Staeck \& al, the strongest predictor of good response of TPE is the presence of epithelial cell foot process fusion and lack of sclerosis on renal biopsy. Early treatment is associated with positive response and it seems prudent to initiate Plasmapheresis as soon as possible after diagnosis of the recurrence of FSGS is made [18]. The association of Rituximab and TPE was described in many studies, and the protocol was well tolerated [5,7]. But we need randomized studies to determine doses and duration of the treatment. The role of cyclosporine A is well established in the stabilization of the podocytes' Actine Cytoskeleton and blocking the production of serum soluble permeability factor by T-Lymphocytes. The dose is $2 \mathrm{mg} / \mathrm{kg}$ intravenous during 14 days with targeted residual serum rates between 200-400 Nano-grams/mL and then move to oral use tablets twice a day [19]. In our patient the protocol followed by the transplantation team is detailed in Figure 6. The French transplantation team in 2017 performed a multicenter retrospective study reports on 19 new cases aged 35 (15-66) years that developed FSGS recurrence at 12 (1.5- 
Citation: Abdellaoui I, Azzabi A, Sahtout W, Benaicha N, Guedri Y, et al. (2019) Rituximab (RTX) and Plasmapheresis in a Recurrent Segmental and Focal Glomerulosclerosis (FSGS) Case after Kidney Transplantation. J Nephrol Ther 9: 333.

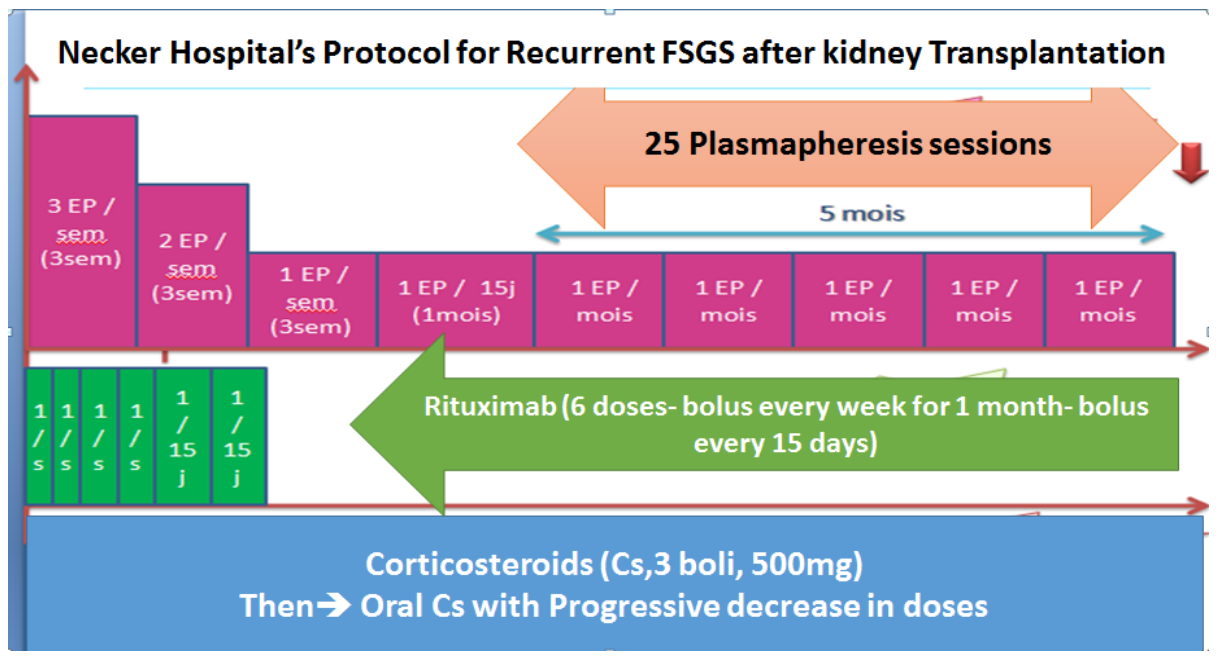

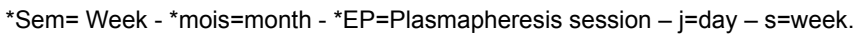

Figure 6: The protocol followed in the case report for Recurrent FSGS after kidney Transplantation.

27) days post-transplantation. Initial treatment consisted of plasma exchanges, high doses of Cyclosporine A and steroids. Rituximab was introduced either immediately or after failure of the initial treatment. Kidney survival at 5 years was $77.4 \%$ (95\% range, 41.9-92.7). The 5 -year graft survival rates in the responding patients and the non-responding patients were $100 \%$ and $36.5 \%$, respectively $(\mathrm{P}=0.01)$. They concluded that rituximab may be a recommended treatment for cases failing either the initial treatment or weaning from Plasmapheresis [20].

Finally, the preventive treatment of recurrent FSGS was described in many studies. In a Korean retrospective study, they reviewed 27 adult renal transplant recipients with FSGS over a period of 10 years. We first compared possible risk factors for FSGS recurrence between the recurrence and non-recurrence groups. Then they evaluated the effect of pretransplant Plasmapheresis $(P P ; n=4)$ and $P P$ with Rituximab (PP+RTX; $=5)$ on recurrence of FSGS after transplantation compared to control patients that were not treated with these modalities. Pretransplant PP or PP+RTX do not significantly decrease the recurrence of FSGS in adult renal transplant candidates [21]. In this case report, the patient didn't receive preventive Plasmapheresis and RTX and the FGGS recurs at day 2 post transplantation. A study comparing recipients with and without preventive treatment seems interesting.

\section{Conclusion}

This study is a simple description of successful treatment using corticosteroid, Cyclosporine A, Plasmapheresis and Rituximab. That protocol obtained complete and sustained remission with normal graft function 9 months after transplantation in a high risk graft recipient exposed to recurrent FSGS. A further study with bigger number of FSGS patients receiving related living donor kidney graft needs to be performed.

\section{References}

1. Crosson JT (2007) Focal segmental glomerulosclerosis and renal transplantation. Transplant Proc 39: 737-43.

2. Kienzl-Wagner K, Rosales A, Scheidl S, Giner T, Bösmüller C (2018) Successful management of recurrent focal segmental glomerulosclerosis. Am J Transplant 18: $2818-2822$

3. Bierzynska A, Saleem MA (2018) Deriving and understanding the risk of post- transplant recurrence of nephrotic syndrome in the light of current molecular and genetic advances. Pediatr Nephrol 33: 2027-2035.

4. Rudnicki M (2016) FSGS Recurrence in Adults after Renal Transplantation BioMed Research International 2016: 7.

5. Alasfar S, Matar D, Montgomery RA, Desai N, Lonze B, et al. (2018) Rituximab and Therapeutic Plasma Exchange in Recurrent Focal Segmental Glomerulosclerosis Post kidney Transplantation. Transplantation 102: e115-120.

6. Kennard AL, Jiang SH, Walters GD (2017) Increased glomerulonephritis recurrence after living related donation. BMC Nephrology 18: 25.

7. Messina M, Gallo E, Mella A, Pagani F, Biancone L (2016) Update on the treatment of focal segmental glomerulosclerosis in renal transplantation. World J Transplant 6: 54-68.

8. Wei C, El Hindi S, Li J, Fornoni A (2011) Circulating urokinase receptor as a cause of focal segmental glomerulosclerosis. Nat Med 17: 952-60.

9. Beaudreuil S, Zhang X, Herr F, Harper F, Candelier JJ, et al. (2019) Circulating CASK is associated with recurrent focal segmental glomerulosclerosis after transplantation. PLoS One 14: e0219353.

10. Delville M, Sigdel TK, Wei C, Li J, Hsieh SC, et al. (2014) A circulating antibody panel for pretransplant prediction of FSGS recurrence after kidney transplantation. Sci Transl Med 6: 256ra136.

11. Amaral S, Neu A (2016) Recurrent FSGS Postkidney Transplant: Moving theNeedle Forward. Clin J Am Soc Nephrol 11: 1932-1934.

12. Bellur SS, Lepeytre F, Vorobyeva O, Troyanov S, Cook HT, et al. (2016) Evidence from the Oxford Classification cohort supports the clinical value of subclassification of focal segmental glomerulosclerosis in $\lg \mathrm{A}$ nephropathy. Kidney Int 91: 235-243

13. D'Agati VD, Kaskel FJ, Falk RJ (2011) Focal Segmental Glomerulosclerosis. N Engl J Med 365: 2398-2311.

14. IJpelaar DTH, Farris AB, Goemaere N, Amann K, Goldschmeding R, et al (2008) Fidelity and Evolution of Recurrent FSGS in Renal Allografts. J Am Soc Nephrol 19: 2219-2224.

15. Fogo $A B$ (2014) Causes and pathogenesis of focal segmenta glomerulosclerosis. Nat Rev Nephrol 11: 76-87.

16. Kashgary A, Sontrop JM, Li L, Al-Jaishi AA, Habibullah ZN, et al. (2016) The role of plasma exchange in treating post-transplant focal segmental glomerulosclerosis: A systematic review and meta-analysis of 77 case-reports and case-series. BMC Nephrol 17: 104.

17. Vlachopanos G, Georgalis A, Gakiopoulou H (2015) Plasma Exchange for the Recurrence of Primary Focal Segmental Glomerulosclerosis in Adult Renal Transplant Recipients: A Meta-Analysis. J Transplant 2015: 639628. 
Citation: Abdellaoui I, Azzabi A, Sahtout W, Benaicha N, Guedri Y, et al. (2019) Rituximab (RTX) and Plasmapheresis in a Recurrent Segmental and Focal Glomerulosclerosis (FSGS) Case after Kidney Transplantation. J Nephrol Ther 9: 333.

18. Staeck O, Slowinski T, Lieker I, Wu K, Rudolph B, et al. (2015) Recurrent Primary Focal Segmental Glomerulosclerosis Managed With Intensified Plasma Exchange and Concomitant Monitoring of Soluble Urokinase-Type Plasminogen Activator Receptor-Mediated Podocyte $\beta 3$-integrin Activation. Transplantation 99: 2593-2597.

19. Beaudreuil S, Lorenzo HK, Elias M, Obada EN, Charpentier B, et al. (2017) Optimal management of primary focal segmental glomerulosclerosis in adults. Int J Nephrol Renovasc Dis 10: 97-107.
20. Garrouste C, Canaud G, Büchler M, Rivalan J, Colosio C, et al. (2017) Rituximab for Recurrence of Primary Focal Segmental Glomerulosclerosis After Kidney Transplantation: Clinical Outcomes. Transplantation 101: 649-56.

21. Park HS, Hong Y, Sun IO, Chung BH, Kim HW, et al. (2014) Effects of pretransplant plasmapheresis and rituximab on recurrence of focal segmental glomerulosclerosis in adult renal transplant recipients. Korean J Intern Med 29: $482-88$. 